\title{
Epidermal barrier maintenance and morphogenesis are molecularly distinct and ceramide synthase 4 (CerS4) is a novel key player in epidermal barrier maintenance
}

Franziska Peters, Frederik Tellkamp, Susanne Brodesser, Emmi Wachsmuth, Bettina Tosetti, Ulrike Karow, Wilhelm Bloch, Olaf Utermöhlen, Martin Krönke, Carien M. Niessen

\section{Introduction \\ The homeostatic maintenance of a functional barrier is crucial for terrestrial life and disturbances in barrier maintenance cause a wide range of common inflammatory diseases. \\ How these barriers are maintained during homeostatic, adult tissue renewal and whether its malfunction drives barrier diseases are still open questions.}

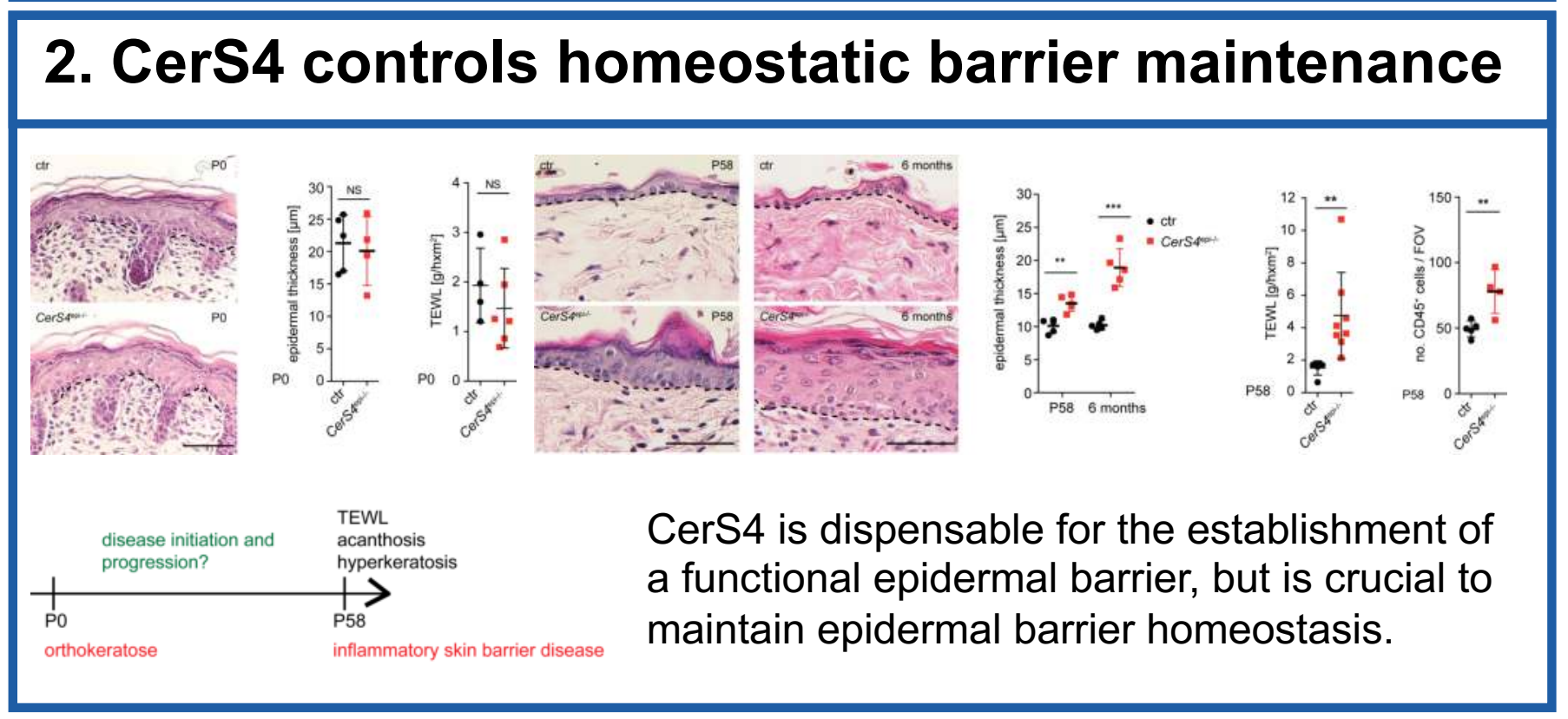

\section{Barrier dysfunction precedes acanthosis and hyperkeratosis}

Epidermal loss of CerS4 triggers a switch from an initially improved to an
impaired barrier function in adult mice, suggesting temporally defined
molecular changes in epidermal barrier composition.

\section{Altered epidermal lipid barrier homeostasis}

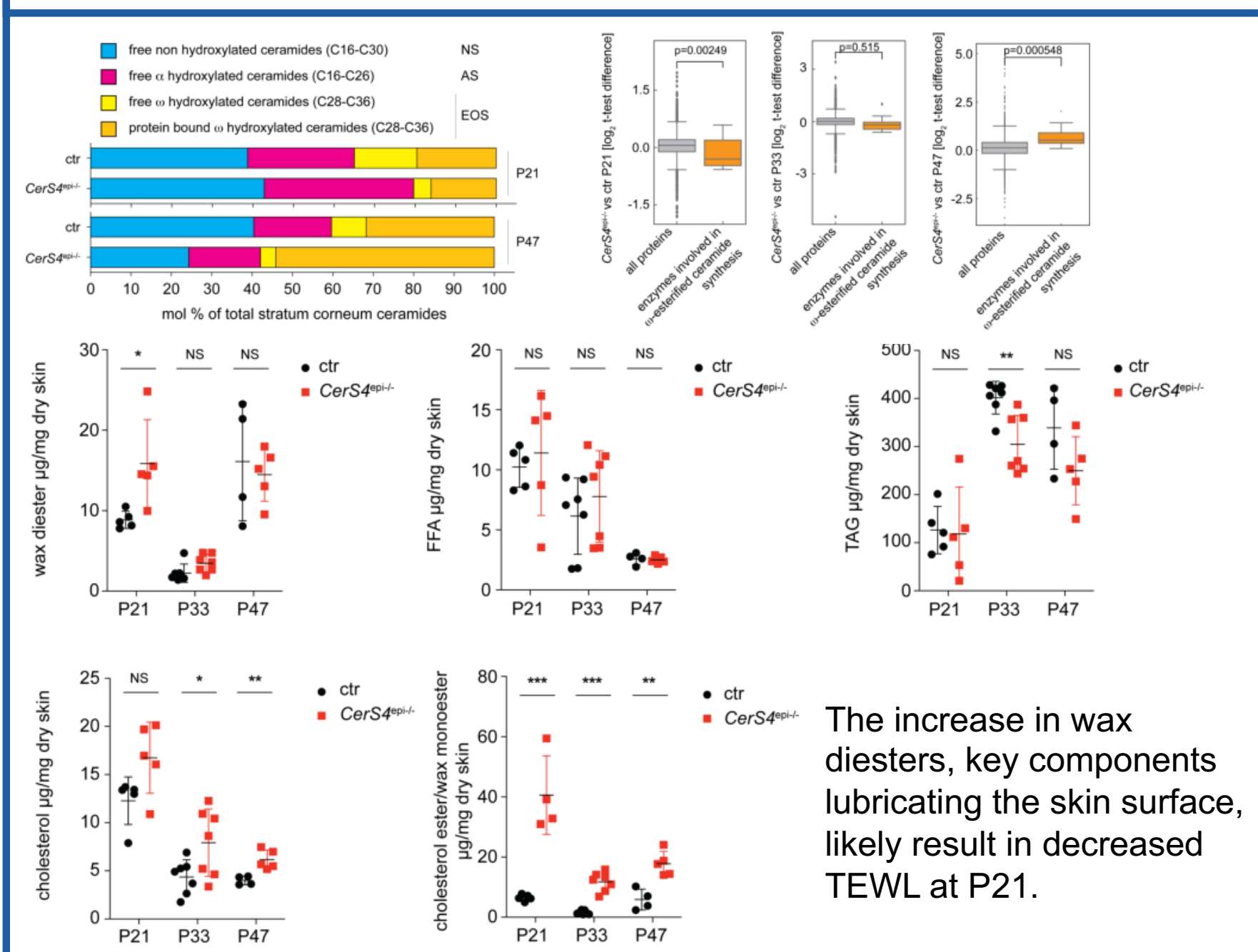

The dysbalance in ceramide composition and the excess of cholesterol chronically disturb the proper lipid packing of the lipid matrix, resulting in impaired barrier function.

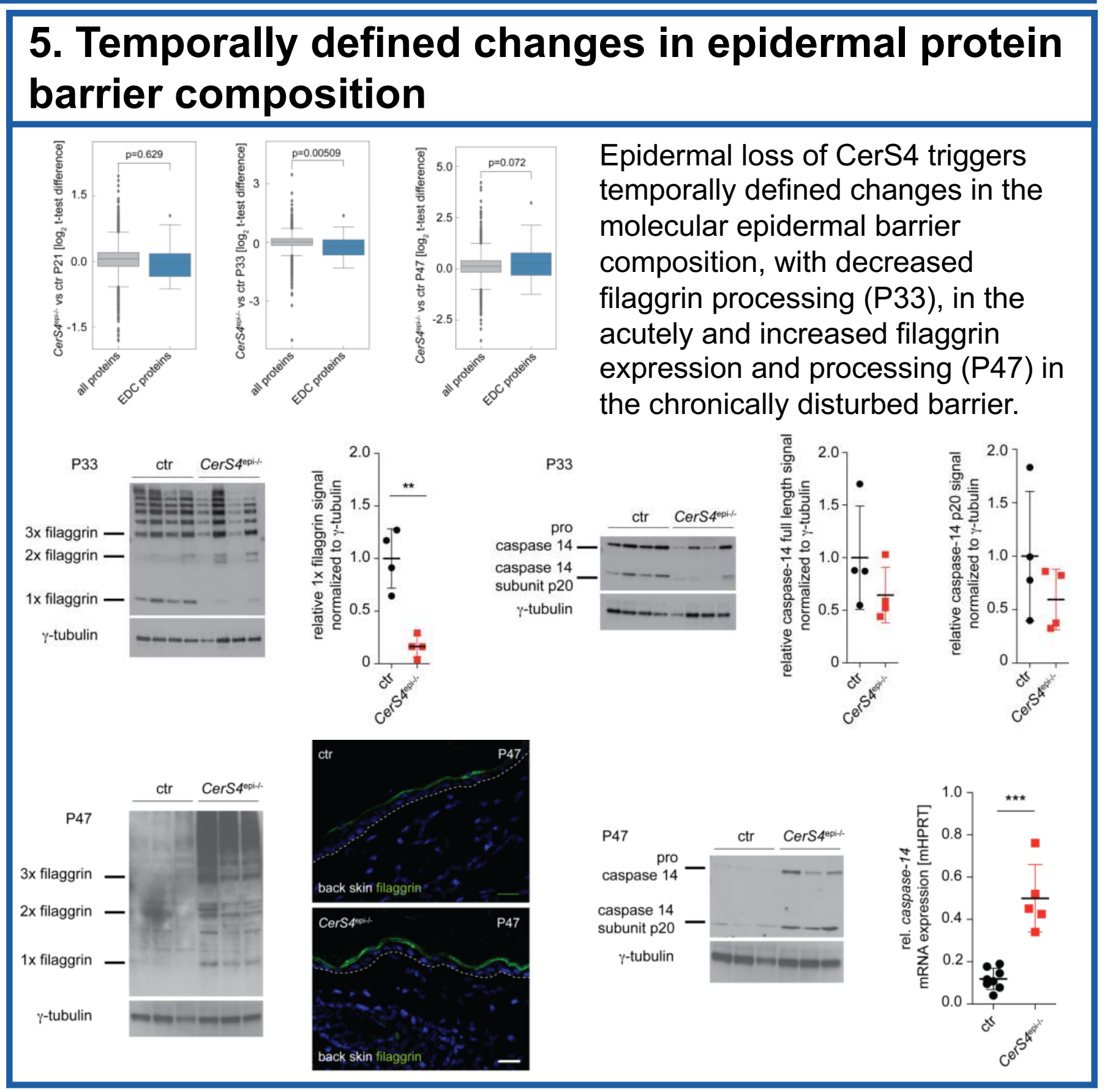

6. Barrier repair response molecularly resembles morphogenetic protein expression signatures

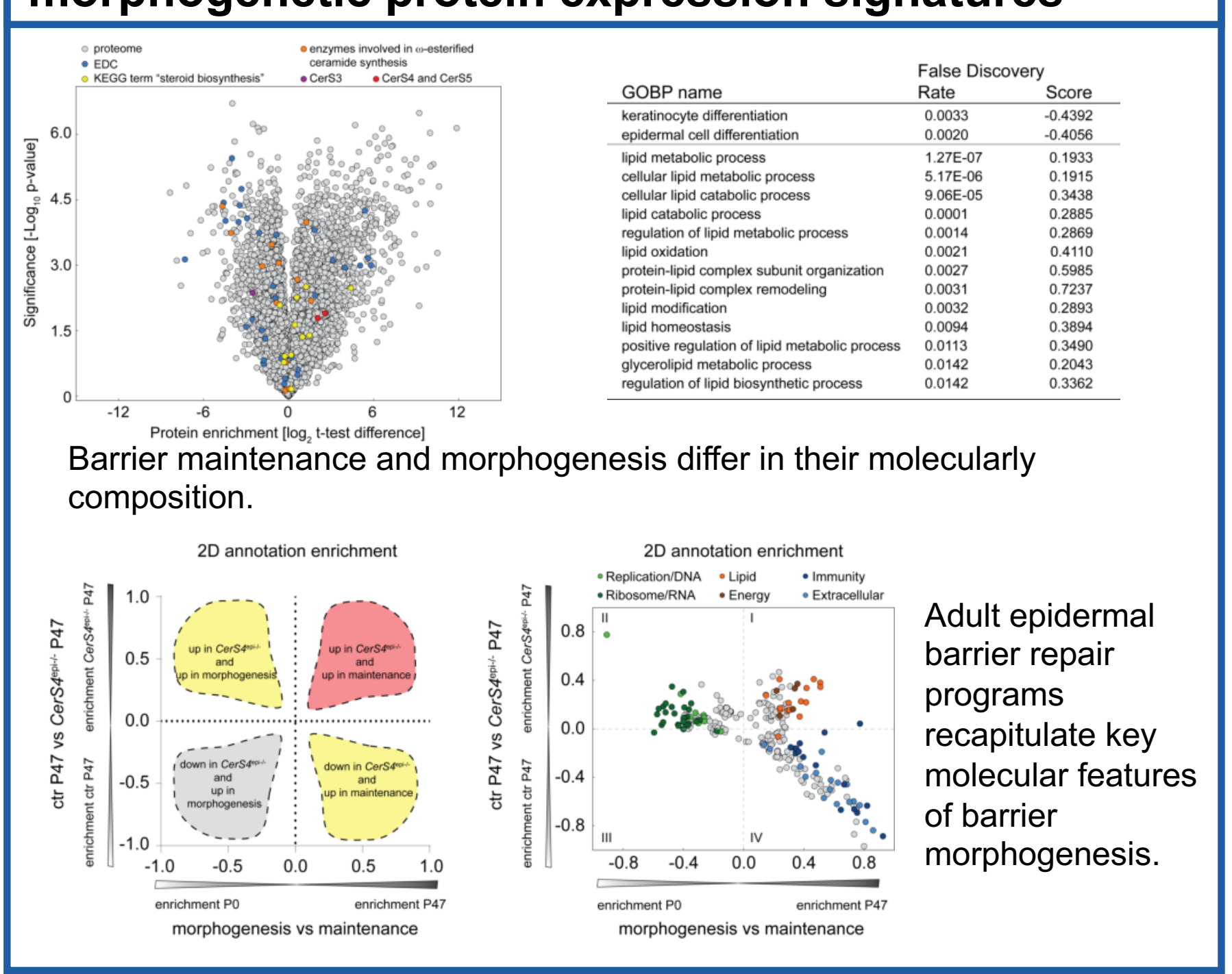

\section{Model}

Altered epidermal ceramide metabolism resulting from epidermal loss of CerS4, triggers temporally defined changes in skin barrier dysfunction, first disturbing epidermal lipid barrier homeostasis, which leads to an impaired epidermal barrier function, ultimately resulting in chronic skin barrier disease.

The data highlight

temporal requirement of specific CerS isoforms in barrier morphogenesis, maintenance, and repair. 